\title{
Effects of talus stabilization taping versus ankle kinesio taping in patients with chronic stroke: a randomized controlled trial
}

\author{
Donghwan Park', Kang-Seong Lee,** \\ 'Department of Physical Therapy, Gyeong-in Medical Rehabilitation Center Hospital, Incheon, Korea \\ ${ }^{2}$ Department of Biomedical Engineering Welfare Technology, Hanseo University, Seosan, Korea
}

Talus stabilization taping (TST) can be utilized to stabilize posteriorly glide of the talus and allows active ankle dorsiflexion movement during walking. The purpose of this study was to compare the effects of ankle kinesio taping with those of TST on ankle dorsiflexion passive range of motion, static balance, the Timed Up and Go test, and the fall risk in patients with chronic stroke and limited ankle dorsiflexion. Participants were randomized into the kinesio taping $(n=11)$ and TST groups $(n=11)$. Both groups were applied the appropriate taping and were asked to walk on the treadmill for $10 \mathrm{~min}$. The ankle dorsiflexion passive range of motion, static balance ability, Timed Up and Go test, and fall risk were assessed in all participants before and after the intervention. The TST group showed greater improvement than the ankle kinesio taping group in all outcomes measured $(P<0.05)$. Furthermore, both groups had significantly decreased in the Timed Up and Go results $(P<0.05)$. This study shows that TST improves functional ability of the ankles of patients with chronic stroke in comparison to the kinesio taping method.

Keywords: Stroke, Ankle range of motion, Balance, Fall, Kinesio taping, Talus stabilization taping

\section{INTRODUCTION}

Stroke patients require long-term treatment and often experience difficulties in daily activities (Carandang et al., 2006). Limited ankle dorsiflexion passive range of motion (DF-PROM) is a common impairment for people with chronic stroke, which leads to impaired balance and increased fall risk in standing or gait (An and Jo, 2017; Kluding and Santos, 2008; Yarkony and Sahgal, 1987). Furthermore, limited ankle DF-PROM results in difficulties in weight transfer toward the paretic lower limb during both standing or gait in patients with stroke (Dickstein et al., 1984; Kluding and Santos, 2008).

Various interventions, such as kinesio taping (KT), nonelastic taping, and talocrural joint mobilization have been used to improve balance ability and gait functions in individual patients with stroke (An and Jo, 2017; Nam et al., 2015; Rojhani-Shirazi et al.,
2015). KT has been used as a rehabilitation program for pain relief, muscle strength enhancement, to correct joint positions, to control joint instability, improve posture alignment, increase proprioception, and to increase range of motion (ROM).

Recent studies have reported that talus stabilization taping (TST) can help restore normal accessory motion in cases of people with limited ankle dorsiflexion ROM (Kang et al., 2014; Yoon et al., 2014). TST provides the posterior talar glide and allows active ankle dorsiflexion movement in a weight-bearing position during walking (Mau and Baker, 2014; Yoon et al., 2014). Evidence on the use of ankle joint mobilization techniques in stroke patients is limited, although researchers have found that talocrural joint mobilization can be useful for improving the balance ability in patients with chronic stroke (An and Jo, 2017) and TST has been recommended to improve ankle DF-PROM and gait functions in cases of people with limited ankle dorsiflexion (Yoon et al.,
${ }^{*}$ Corresponding author: Kang-Seong Lee (iD https://orcid.org/0000-0002-8616-0106 Department of Biomedical Engineering Welfare Technology, Hanseo University, 46 Hanseo 1-ro, Haemi-Myun, Seosan 31962, Korea

E-mail: cpo114@hanseo.ac.kr

Received: October 18, 2019 / Accepted: November 12, 2019
This is an Open Access article distributed under the terms of the Creative Commons Attribution Non-Commercial License (http://creativecommons.org/licenses/by-nc/4.0/) which permits unrestricted non-commercial use, distribution, and reproduction in any medium, provided the original work is properly cited. 
2014).

Although TST increases ankle DF-PROM and weight shift ability in individuals with limited ankle dorsiflexion, to date it appears that the usefulness of TST in patients with chronic stroke has not been investigated. Therefore, the purpose of this study is to compare the immediate effects of a TST with those of ankle KT on ankle DF-PROM, static balance ability, Timed Up and Go (TUG) test, and fall risk in patients with chronic stroke.

\section{MATERIALS AND METHODS}

\section{Subjects}

A pilot test was performed on four volunteers to determine the number of chronic stroke patients required for this study. A power analysis was performed using the G-power software (G-power software 3.1.2; Franz Faul, University of Kiel, Kiel, Germany) to achieve a significant power of 0.80 , the effect size of 1.26 , and $\alpha$ level of 0.05 . The results of the power analysis showed that this study would require nine patients with chronic stroke per group. The present study involved 22 patients with stroke from Youngdungpo hospital, Seoul who volunteered to participate in this study. The inclusion criteria were as follows: (a) diagnosis of hemiplegia due to hemorrhagic or ischemic stroke ( $>6$ months poststroke); (b) independent gait possible over $10 \mathrm{~m}$ without assistive devices; (c) minimum score of 24 on the Korean Mini-Mental State Examination; (d) spasticity in a lower affected side ankle (modified Ashworth scale $\geq 1$ ), (e) less than $8^{\circ}$ of ankle DFPROM on the affected side. The exclusion criteria were a history of surgery in the lower extremities, fractures, hip and knee flexion contracture and contraindications for ankle joint mobilization. Before the study, all participants were informed of the purpose and procedure of the study, in accordance with the ethical standards of the Declaration of Helsinki. Only those who voluntarily signed informed consent form were enrolled as subjects.

\section{Experimental procedure}

A randomized controlled study design was used. Participants were randomly assigned to either the ankle KT $(n=11)$ or TST $(\mathrm{n}=11)$ group. The participant characteristics and outcomes (ankle DF-PROM, static balance ability, TUG test, and fall risk) measures were assessed by two examiners who were blinded to the task of participant intervention and study hypotheses. Before measuring the baseline data, participants rested in a chair for at least $5 \mathrm{~min}$. The ankle KT and TST were applied $10 \mathrm{~min}$ after the baseline measurements were made. Another experienced KT certified physical therapist applied two types of taping to the affected side ankle. Before the tape application, the skin surface was removed of hair and cleansed. For the ankle KT used standard $5 \mathrm{~cm}$ 3NS TEX (TS Co., LTD, Seoul, Korea) was used and applied in accordance with Kase's methods (Yazici et al., 2015). The first taping began from the dorsum of the foot and was attached to the anterior aspect of the tibia. The second taping was attached with the maximum length of the tape, about $10 \mathrm{~cm}$ above the lateral malleolus from the sole of the foot. The third taping began from the anterior of the ankle and was attached to the posterior aspect of the ankle with the maximum length of the tape from the lateral side of the ankle (Fig. 1A). The ankle TST was applied with rigid strapping tape (37-mm width, Mueller $\mathrm{M}$ tape) using maximal force with
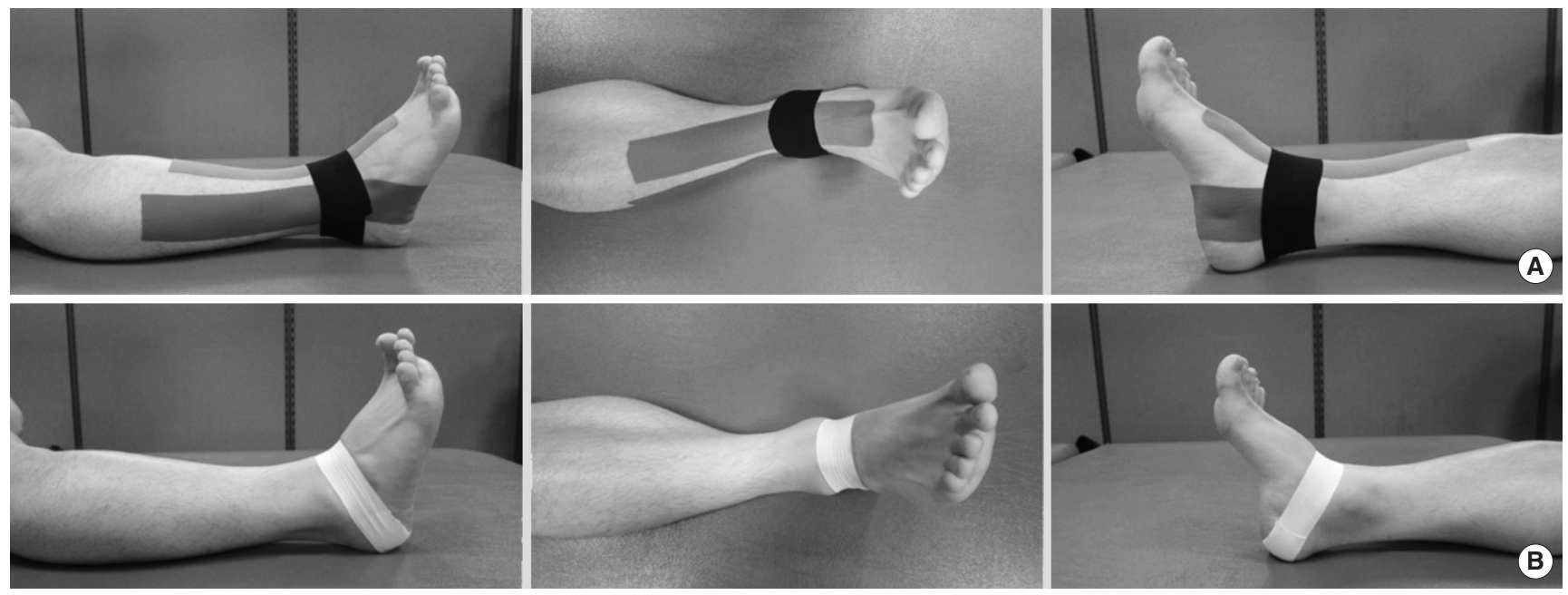

Fig. 1. Application of ankle kinesio taping method (A) and talus-stabilizing taping method (B). 
the piece of tape. Each participant placed the ankle of the affected side on the chair and advanced the tibia over the foot via knee flexion, then, the tape was attached to the plantar surface of the calcaneus through the medial and lateral sides of the talus (Yoon et al., 2014) (Fig. 1B). After applying each of the two taping types, the participants performed a walk at a comfortable speed on the treadmill for $10 \mathrm{~min}$ to familiarize themselves with the two conditions. Immediately following the removal of the tape after the treadmill walking, a 5-min resting period was allowed and the post data collection began.

\section{Outcome measurements}

Dependent variables were as follows: ankle DF-PROM, static balance ability, TUG test, and fall risk. Ankle DF-PROM was measured using a $20-\mathrm{cm}$ standard goniometer after flex of the knee to $90^{\circ}$ in the prone position. The central axis of the goniometer was placed on the lateral malleolus. The stationary arm of the goniometer was placed parallel to the lateral side of the fifth metatarsal bone and the moving arm was placed parallel to the center of the fibular head. One examiner applied force to the plantar surface of the forefoot and midfoot in the direction of dorsiflexion until further movement was firmly restricted. A second examiner confirmed the ankle DF-PROM. The static balance ability and fall risk were measured using the Biodex Balance System SD (Biodex Medical Systems, Inc., Shirley, NY, USA) that enables the device to serve as an objective assessment of balance ability. This system uses a circular platform which is free to move in the anteriorposterior and medial-lateral axes simultaneously and measures the degree of tilt in each axis, providing an average sway score. For the static balance ability measures, the stability index is measured as the angular excursion of a patient's center of gravity and measurements were run for $30 \mathrm{sec}$. For fall risk measures, the results included an overall stability index score. Based on the results of the previous study (Cakar et al., 2010), level 12 was chosen as the appropriate level to perform for all test participants and was performed for $20 \mathrm{sec}$. A high score indicates a lot of movement and poor balance. The TUG test records the time taken to stand up from a $50-\mathrm{cm}$ height chair, walk $3 \mathrm{~m}$ and turn around a block, walk back to the chair, and sit down. All measurements were repeated 3 times, and the mean value was calculated.

\section{Statistical analysis}

PASW Statistics 18 software (SPSS, Chicago, IL, USA) was used for all statistical analyses. Normality of the data distribution was examined using the one-sample Kolmogorov-Smirnov test.
Data are presented as mean \pm standard deviation. Baseline demographic variables were compared between the groups using an independent $t$-test for continuous data and the chi-square test of independence for categorical data. After the intervention, independent $t$-tests were used to compare between-group means, and paired $t$-tests were used to compare within-group means. The effect sizes were calculated to determine meaningful changes between the groups; an effect size of $P \leq 0.20$ indicates a small change; 0.50 a moderate change; and 0.80 a large change (Cohen, 1992). Statistical significance was set at $P$ values of $<0.05$.

\section{RESULTS}

Physical characteristics are summarized in Table 1. Outcome measurements are presented in Table 2. No significant differences were observed between the groups in any of the measured baseline values. Following interventions, ankle DF-PROM was significantly increased in the TST group compared to the KT group $(P<0.001)$ and was also significantly increased relative to baseline values only in the TST group $(P<0.001)$. Similarly, static balance ability was significantly decreased in the TST group compared to the KT group $(P<0.001)$ and significantly decreased relative to baseline values only in the TST group $(P<0.001)$. Additionally, the TUG results were significantly decreased in the TST group relative to the KT group $(P<0.001)$. TUG decreased significantly compared to baseline values in both the KT $(P=0.012)$ and TST group $(P<0.001)$. Further, fall risk was significantly decreased in the TST group compared to the KT group $(P<0.001)$ and was significantly decreased relative to baseline values in only the TST group $(P<0.001)$.

Table 1. Clinical information of the patients with stroke

\begin{tabular}{lccc}
\hline Characteristic & $\begin{array}{c}\text { Kinesio taping } \\
(\mathrm{n}=11)\end{array}$ & $\begin{array}{c}\text { Talus } \\
\text { stabilization } \\
\text { taping }(\mathrm{n}=11)\end{array}$ & P-value \\
\hline Age $(\mathrm{yr})$ & $60.09 \pm 4.50$ & $59.9 \pm 6.49$ & 0.940 \\
Height $(\mathrm{cm})$ & $167.45 \pm 8.04$ & $165.55 \pm 7.06$ & 0.561 \\
Weight $(\mathrm{kg})$ & $64.27 \pm 9.49$ & $65.45 \pm 9.61$ & 0.775 \\
Sex, male:female & $8: 3$ & $7: 4$ & 0.647 \\
Hemiplegic side, left:right & $5: 6$ & $4: 7$ & 0.665 \\
Type of stroke, ischemia:hemorrhag & $4: 7$ & $6: 5$ & 0.392 \\
Disease duration (month) & $10.27 \pm 2.72$ & $9.82 \pm 2.48$ & 0.687 \\
K-MMSE & $26.09 \pm 2.17$ & $26.27 \pm 2.15$ & 0.845 \\
\hline
\end{tabular}

Values are presented as mean \pm standard deviation or number. K-MMSE, Korean version of Mini-Mental State Examination. 
Table 2. Changes in the intervention in each group

\begin{tabular}{|c|c|c|c|c|c|c|c|c|}
\hline \multirow{2}{*}{ Parameter } & \multicolumn{4}{|c|}{ Kinesio taping $(n=11)$} & \multicolumn{4}{|c|}{ Talus stabilization taping $(\mathrm{n}=11)$} \\
\hline & Pretest & Posttest & Change score (CI) & Effect size & Pretest & Posttest & Change score (CI) & Effect size \\
\hline DF-PROM $\left(^{\circ}\right)$ & $6.81 \pm 0.77$ & $7.26 \pm 0.94$ & $0.45(0.16-1.05)$ & 0.53 & $6.18 \pm 0.97$ & $9.91 \pm 1.34^{*}$ & $3.73(3.09-4.37)^{\dagger}$ & 3.22 \\
\hline Static balance ability result (score) & $0.9 \pm 0.18$ & $0.82 \pm 0.15$ & $-0.08(-0.19$ to 0.03$)$ & 0.48 & $0.95 \pm 0.24$ & $0.55 \pm 0.13^{*}$ & $-0.39(-0.24 \text { to } 0.54)^{\dagger}$ & 2.16 \\
\hline Timed Up and Go test results (score) & $21.88 \pm 2.45$ & $20.49 \pm 2.87$ & $-1.4(-2.41 \text { to }-0.38)^{*}$ & 0.52 & $22.54 \pm 2.94$ & $16.43 \pm 2.21^{*}$ & $-6.11(-5.08 \text { to }-7.14)^{\dagger}$ & 2.37 \\
\hline Fall risk (point) & $3.78 \pm 0.32$ & $3.65 \pm 0.46$ & $-0.13(-0.4$ to 0.15$)$ & 0.33 & $3.89 \pm 0.32$ & $2.77 \pm 0.46^{*}$ & $-1.12(-1.38 \text { to }-0.85)^{\dagger}$ & 2.87 \\
\hline
\end{tabular}

Values are expressed as mean \pm standard deviation.

DF-PROM, dorsiflexion passive range of motion; $\mathrm{Cl}$, confidence interval.

Change score $=$ posttest-pretest .

${ }^{*} P<0.05$, significant difference between pre- and postinterventions within the group. ${ }^{\dagger} P<0.05$, significant difference between the change scores between the groups.

\section{DISCUSSION}

The objective of this study was to compare the immediate efficacy of TST and ankle KT therapy in improving ankle DFPROM, SBA, TUG test results, and fall risk in chronic stroke patients. The results indicated that the TST group demonstrated significant improvements in all of the measured parameters in comparison with baseline values while the KT group demonstrated significant improvement only in the TUG test result. The TST group demonstrated significantly improved results in ankle DFPROM, static balance ability, the TUG result, and fall risk in comparison to the KT group following completion of the intervention. To the best of our knowledge, this is the first investigation to demonstrate both a TST intervention benefit and to compare the differential effects of TST and ankle KT in chronic stroke patients.

Compared with the KT group, ankle DF-PROM significantly increased in the TST group by $53.5 \%$ after study intervention. Additionally, in the TST group, the DF-PROM significantly increased by $37.6 \%$ after the TST intervention compared with baseline. These results are consistent with those of previous studies (An and Jo, 2017; Kang et al., 2014). An and Jo (2017) reported significant improvements in ankle DF-PROM, ankle strength, and weight-bearing ability following talocrural mobilization with movement application in patients with chronic stroke. Another recent study showed that ankle DF-PROM and heel-off time in the stance phase of gait were increased after walking with TST application in people with ankle limited DF-PROM (Kang et al., 2014). Their ankle DF-PROM results were similar to those in the present study. Here, a weight-bearing TST facilitated the recovery of accessory movement in the ankle joint by the application of a gliding force to the talus posteriorly and may have enhanced the stretch force in the ankle plantar flexors during the repeated DFROM walking (Kang et al., 2014; Vicenzino et al., 2006). In ad- dition, the recovery of posterior talar glide contributes to the improvement of ankle dorsiflexion ROM (Vicenzino et al., 2006). For this reason, TST also seems to increase ankle DF-PROM compared to KT. Thus, our results indicate that TST effectively increases ankle DF-PROM.

After the completion of the study interventions, ankle static balance ability decreased in the TST group by $78.9 \%$ compared to the KT group. Additionally, in the TST group, the static balance ability significantly decreased by $72.7 \%$ after the TST intervention compared with baseline. These results are consistent with those of previous studies (Kim and Lee, 2018; Park et al., 2019). Kim and Lee (2018) reported significant improvements in ankle ROM, static balance ability, TUG test, and dynamic gait index following weight-bearing-based mobilization with movement application in patients with stroke. Park et al. (2019) applied ankle mobilization with movement in patients with chronic stroke, which produced significant improvements in static balance ability, Berg balance scale, and cadence compared with static calf muscle stretching. In our study, static balance ability significantly improved in the TST group compared to the KT group. Joint mobilization is effectively helping the activity of mechanoreceptors in the capsule and ligaments, thereby improving ankle sensory output (Hiller et al., 2006). Also, possible restoration of joint arthrokinematics after walking with TST could explain the joint position sense and postural control by changing the muscle activity around the ankle joint (Bae et al., 2015; Franettovich et al., 2008). For this reason, TST also appears to improve static balance ability compared to KT.

After the trial protocol, the TUG results significantly decreased in the TST group by $77.1 \%$ compared with the KT group. Further, both groups demonstrated significant improvements in the TUG results following interventions, decreasing by $6.8 \%$ in the KT group and $37.2 \%$ in the TST group. These results are consistent with those of previous studies (Lee et al., 2017; Sheng et al., 2019). 
Sheng et al. (2019) demonstrated that ankle KT intervention significantly improved 10-m walking test, TUG results, stride length, stance phase, and swing phase compared with baseline in patients with foot drop after stroke. Specifically, posterior talar glide with dorsiflexion ankle joint mobilization was shown to significantly increase ankle ROM, ankle strength, TUG results, and gait function compared with the placebo group in patients with stroke (Lee et al., 2017). Ankle KT might reduce the recruitment time to the dynamic ankle joint stabilizer muscles, ensure the proper posture of the foot, and improve the contact of the foot with the ground (Morris et al., 2013; Yazici et al., 2015). In the present study, TST may also improve postural control, ankle stability and proper foot positioning by the response of the ankle movement as was also shown in KT. In addition, TST is used to glide the talus posteriorly and allow ankle dorsiflexion during walking. In these procedures, the foot was restored to normal ankle joint kinematics. This may have provided a greater stability force for the talus than KT does. As a result, TST also seems to improve TUG results compared to KT.

Compared with the KT group, fall risk significantly decreased in the TST group by $88.4 \%$ after intervention and additionally decreased by $40.4 \%$ after the TST intervention compared with baseline. These results are consistent with those of previous studies (Mackintosh et al., 2006; Mecagni et al., 2000). Mackintosh et al. (2006) investigated the predictors associated falls poststroke and found that reduced balance ability, slower walking speed, and lack of activity may increase instances of falls and that balance and functional mobility training were needed. Mecagni et al. (2000) compared the relationship between balance measures and ankle $\mathrm{ROM}$ in community-dwelling elderly women and found that a relationship exists between ankle ROM and balance scores (0.29 to 0.63 .) and these results may indicate that ankle ROM contributes to the maintenance of balance. Their fall risk results were similar to those in the present study. Patients with stroke have shifted body weight in the less affected lower extremities and this asymmetrical posture leads to a decrease in balance ability and increase fall risk (Cakar et al., 2010; Dickstein et al., 1984). In the present investigation, we suggest that walking with TST may improve ankle DF-PROM along with static and dynamic balance ability which may contribute to improvements in the fall risk by improving postural sway and proper foot position in the ankle joint when standing up. Also, TST under the weight-bearing condition may have enhanced the stretch force in the ankle plantar flexors. Interventions to improve ankle ROM and balance may reduce the fall risk. For this reason, TST also seems to improve fall risk compared to KT. Thus, our results indicate that TST effectively improves fall risk.

This study has several limitations. Firstly, this study did not include follow-up tests; therefore, the carryover effect of TST was not studied. Additional studies are needed to elucidate these effects. Secondly, this study measured ankle ROM only passively. Follow-up studies are needed to determine the changes in ankle ROM during functional activities. Finally, findings may not be generalized for all stroke patients, as our study was studied on stroke patients with minor or moderate physical impairment. Additional studies are required to study these specific issues in more detail in the future. This study compared the effects of ankle KT and TST with respect to ankle DF-PROM, static balance ability, TUG test results, and fall risk in patients with chronic stroke. Our findings indicate that TST significantly improves ankle DFPROM, static balance ability, TUG test results, and fall risk compared to the ankle KT group. In addition, both ankle KT and TST interventions significantly improved the TUG test results. Taken together, these findings will be the basis for the development of novel therapeutic interventions and strategies for people with chronic stroke.

\section{CONFLICT OF INTEREST}

No potential conflict of interest relevant to this article was reported.

\section{REFERENCES}

An CM, Jo SO. Effects of talocrural mobilization with movement on ankle strength, mobility, and weight-bearing ability in hemiplegic patients with chronic stroke: a randomized controlled trial. J Stroke Cerebrovasc Dis 2017;26:169-176.

Bae YH, Kim HG, Min KS, Lee SM. Effects of lower-leg kinesiology taping on balance ability in stroke patients with foot drop. Evid Based Complement Alternat Med 2015;2015:125629.

Cakar E, Durmus O, Tekin L, Dincer U, Kiralp MZ. The ankle-foot orthosis improves balance and reduces fall risk of chronic spastic hemiparetic patients. Eur J Phys Rehabil Med 2010;46:363-368.

Carandang R, Seshadri S, Beiser A, Kelly-Hayes M, Kase CS, Kannel WB, Wolf PA. Trends in incidence, lifetime risk, severity, and 30-day mortality of stroke over the past 50 years. JAMA 2006;296:2939-2946.

Cohen J. Statistical power analysis. Curr Dir Psychol Sci 1992;1:98-101.

Dickstein R, Nissan M, Pillar T, Scheer D. Foot-ground pressure pattern of standing hemiplegic patients. Major characteristics and patterns of 
improvement. Phys Ther 1984;64:19-23.

Franettovich M, Chapman A, Blanch P, Vicenzino B. A physiological and psychological basis for anti-pronation taping from a critical review of the literature. Sports Med 2008;38:617-631.

Hiller CE, Refshauge KM, Bundy AC, Herbert RD, Kilbreath SL. The Cumberland ankle instability tool: a report of validity and reliability testing. Arch Phys Med Rehabil 2006;87:1235-1241.

Kang MH, Kim JW, Choung SD, Park KN, Kwon OY, Oh JS. Immediate effect of walking with talus-stabilizing taping on ankle kinematics in subjects with limited ankle dorsiflexion. Phys Ther Sport 2014;15:156161.

Kim SL, Lee BH. The effects of posterior talar glide and dorsiflexion of the ankle plus mobilization with movement on balance and gait function in patient with chronic stroke: a randomized controlled trial. J Neurosci Rural Pract 2018;9:61-67.

Kluding PM, Santos M. Effects of ankle joint mobilizations in adults poststroke: a pilot study. Arch Phys Med Rehabil 2008;89:449-456.

Lee J, Kim JO, Lee BH. The effects of posterior talar glide with dorsiflexion of the ankle on mobility, muscle strength and balance in stroke patients: a randomised controlled trial. J Phys Ther Sci 2017;29:452-456.

Mackintosh SF, Hill KD, Dodd KJ, Goldie PA, Culham EG. Balance score and a history of falls in hospital predict recurrent falls in the 6 months following stroke rehabilitation. Arch Phys Med Rehabil 2006;87:15831589.

Mau H, Baker RT. A modified mobilization-with-movement to treat a lateral ankle sprain. Int J Sports Phys Ther 2014;9:540-548.

Mecagni C, Smith JP, Roberts KE, O'Sullivan SB. Balance and ankle range of motion in community-dwelling women aged 64 to 87 years: a correlational study. Phys Ther 2000;80:1004-1011.
Morris D, Jones D, Ryan H, Ryan CG. The clinical effects of Kinesio ${ }^{\circledR}$ Tex taping: a systematic review. Physiother Theory Pract 2013;29:259-270.

$\mathrm{Nam} \mathrm{CW}$, Lee JH, Cho SH. The effect of non-elastic taping on balance and gait function in patients with stroke. J Phys Ther Sci 2015;27:2857-2860.

Park D, Lee JH, Kang TW, Cynn HS. Four-week training involving ankle mobilization with movement versus static muscle stretching in patients with chronic stroke: a randomized controlled trial. Top Stroke Rehabil 2019;26:81-86.

Rojhani-Shirazi Z, Amirian S, Meftahi N. Effects of ankle kinesio taping on postural control in stroke patients. J Stroke Cerebrovasc Dis 2015; 24:2565-2571.

Sheng Y, Kan S, Wen Z, Chen W, Qi Q, Qu Q, Yu B. Effect of kinesio taping on the walking ability of patients with foot drop after stroke. Evid Based Complement Alternat Med 2019;2019:2459852.

Vicenzino B, Branjerdporn M, Teys P, Jordan K. Initial changes in posterior talar glide and dorsiflexion of the ankle after mobilization with movement in individuals with recurrent ankle sprain. J Orthop Sports Phys Ther 2006;36:464-471.

Yarkony GM, Sahgal V. Contractures. A major complication of craniocerebral trauma. Clin Orthop Relat Res 1987;(219):93-96.

Yazici G, Guclu-Gunduz A, Bayraktar D, Aksoy S, Nazliel B, Kilinc M, Yildirim SA, Irkec C. Does correcting position and increasing sensorial input of the foot and ankle with Kinesio Taping improve balance in stroke patients? NeuroRehabilitation 2015;36:345-353.

Yoon JY, Hwang YI, An DH, Oh JS. Changes in kinetic, kinematic, and temporal parameters of walking in people with limited ankle dorsiflexion: pre-post application of modified mobilization with movement using talus glide taping. J Manipulative Physiol Ther 2014;37:320-325. 